\title{
Deep Conservation in Urban India and its Implications for the Design of Conservation Technologies
}

\author{
Yedendra B. Shrinivasan*, Mohit Jain*, Deva P. Seetharam*, Abhishek Choudhary*, \\ Elaine Huang ${ }^{\#}$, Tawanna Dillahunt ${ }^{+}$, Jennifer Mankoff ${ }^{+}$ \\ *IBM Research \\ Bangalore, India \\ \{yshriniv, mojain13, \\ dseetharam\}@in.ibm.com
}

\begin{abstract}
Rapid depletion of fossil fuels and water resources has become an international problem. Urban residential households are among the primary consumers of resources and are deeply affected by resource shortages. Despite the global nature of these problems, most of the solutions being developed to address these issues are based on studies done in the developed world. We present a study of energy, water and fuel conservation practices in urban India. Our study highlights a culture of deep conservation and the results raise questions about the viability of typical solutions such as home energy monitors. We identify new opportunities for design such as point-of-use feedback technologies, modular solutions, distributed energy storage, harnessing by-products and automated load shifting.
\end{abstract}

\section{Author Keywords \\ Energy; Sustainability; Developing World.}

\section{ACM Classification Keywords}

H.5.m. Information interfaces and presentation (e.g., HCI): Miscellaneous.

\section{INTRODUCTION}

Global consumption of water and energy has been increasing rapidly over the past decades, and both resources are in short supply. While increased supply is one solution to this problem, this cannot always be done fast enough to meet demand, and some resources (such as fossil fuels and groundwater) cannot be replaced as they cannot be quickly replenished. An alternative solution is to decrease demand and wastage. This requires a deep understanding of consumption practices; attitudes towards conservation; and other factors affecting resource use. Several studies, primarily in residential settings, provide insight into these factors and their potential implications for technology design $[11,15,25,36,44,45]$. However, most have focused on developed countries, primarily in North

Permission to make digital or hard copies of all or part of this work for personal or classroom use is granted without fee provided that copies are not made or distributed for profit or commercial advantage and that copies bear this notice and the full citation on the first page. To copy otherwise, or republish, to post on servers or to redistribute to lists, requires prior specific permission and/or a fee.

CHI 2013, April 27-May 2, 2013, Paris, France.

Copyright (C) 2013 ACM 978-1-4503-1899-0/13/04..\$15.00.
America. As a result, there is little information about whether or how those results might be applicable to other geographies, cultures, and socioeconomic groups.

About $82 \%$ of the world's population is in developing nations [47] and by 2020 the developing world is projected to account for $40 \%$ of the global energy use [37]. Hence, there is great value in understanding what drives resource consumption and conservation in the developing world. In this paper, we present a study of energy, fuel and water management practices in urban India, focusing primarily on affluent households in Bangalore. We chose to focus on middle and high-income households because they consume resources in more diverse ways (e.g., own multiple types of appliances). Our focus on Bangalore allowed us to gather data from "early adopters" that might be using technologies likely to become widespread in the future.

India is the world's second most populous country, with a population of over 1.2 billion in 2013 [9]. India is growing at a rate of about $1.3 \%$ per year and economic growth has been averaging more than 7\% per year since 1997 [9]. These two trends have led to a substantial increase in national consumption of energy [18] and water [19]. The Indian electricity sector produces 880 billion $\mathrm{kWh}$ per year (the world's $7^{\text {th }}$ largest production) [9], while about 400 million Indians have no access to electricity [46] and about 80 million lack access to clean water ${ }^{1}$ [42], due to a combination of poverty and infrastructure limitations. Even those who have access to resources face severe supply shortages. As late as 2007, 20 major Indian cities provided clean supply for only 4.3 hours per day, and no city supplied continuous clean water [6]. Similarly, households with electricity connections face several hours of power cuts per day due to an $8.9 \%(7.5 \mathrm{GWh})$ energy shortage [8].

Urban India constitutes a major share of Indian energy consumption (about 39\% of energy use [7]). Domestic water use is only 5\% nationally in 2000 [4] (a smaller number than energy because most water is used for agriculture and industry). It is projected to increase to $8 \%$ by 2025 , of which urban water use will be $74 \%$ (urban and

\footnotetext{
1 According to Indian norms, access means at least 40 liters/capita/day of safe drinking water available within a distance of $\sim 1.6 \mathrm{~km}$ [42].
} 

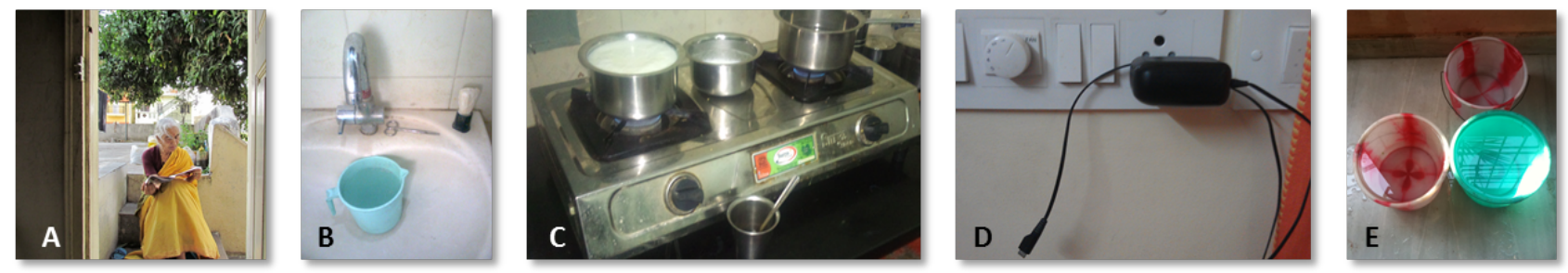

Figure 1. Examples of "extreme" conservation actions (when compared to typical practices in the developed world) (A) Sitting outside to use natural light: "it's like where you place yourself for reading. That's what matters." (Bala) (B) Water conservation is built into daily activities: "I use like two mugs for shaving, it's a routine thing... so no runoff of water." (Raj) (C) The middle pot is using the

dissipated heat of the left and right burner "... also try to use the dissipated heat... trying to be very efficient... you're also trying to optimize your time." (John). (D) Every device, large or small, has a switch, and nothing is left on (except the refrigerator) (E) Water re-use (multiple times) and storage (against shortages) is done using these typical buckets (photo taken by Kumar).

rural: 200 and 70 liters per capita per day respectively) [4]. A majority of urban households have access to electricity [3] and water [31] (91\% and $80 \%$ respectively). An understanding of consumption and conservation practices in urban Indian households is crucial for designing appropriate technologies for this population.

To address this gap, we conducted a photo-elicitation study in urban India (primarily, Bangalore). Our findings illustrate a practice of deep conservation. Some factors that influence conservation behavior included motivations such as resource scarcity and household characteristics such as the use of household staff. Figure 1 shows a few examples: reading outdoors to reduce the use of electric lighting; heating milk by placing it between two burners of a stove to benefit from "dissipated heat"; storing water in buckets for later use and re-use.

Our primary contribution is a rich description of everyday practice around resource management, including water, electricity and fuels, in urban households in India. We also provide design implications showing value in point-of-use feedback rather than monitoring technologies, modular solutions, distributed energy storage, harnessing byproducts and automated load shifting. Although these implications are derived from a particular population in India, they may inform work in other regions with similar characteristics such as scarce resources or multiple household staff (common in many countries where cheap household labor is available).

\section{RELATED WORK}

This section starts by reviewing some of the key results in the developed world around energy and water use [e.g., 1, $5,11,15,23,35,36]$ and their findings about conservation practices, motivations and challenges. This is followed by relevant work from the developing world [13, 25, 29, 33], with a particular focus on India.

\section{Conservation in the Developed World}

A number of articles have explored energy conservation behaviors and attitudes in developed countries, including Australia [29], UK [27], and in the U.S. green households [45], typical middle-income households [11, 36], and lowincome households [15]. Some of the themes highlighted in this body of work include reasons for saving energy (such as future generations, spirituality, ethics, habit, and trends), approaches to saving energy (such as repair work, re-use, efficient purchases and monitoring), and barriers to saving energy (such as money, safety, other household members and infrastructure inefficiencies). Other studies of routine domestic energy consumption practices in U.S. households and their interactions with specific devices [36] find that most energy consumption interactions become unconscious, habitual and in some cases, irrational $[36,40]$.

There is also a long history of interventions designed to reduce energy use and/or encourage other types of green behavior (such as recycling) in the developed world, as summarized in [1]. Within the CHI community, a number of papers have been published on interaction designs for eco-visualization and feedback (e.g., [23, 34, 35]).

Although most of this past work has focused on energy, Chetty et al. [11] discuss water conservation strategies such as taking shorter showers and doing dishes by hand instead of with a dishwasher. Researchers have also developed mechanisms for sensing water consumption at fixture level (e.g., $[5,20,21,26])$. Water-related eco-feedback has been provided using ambient displays at the point-ofconsumption in both public [5] and private spaces [26], and in-home displays showing the aggregate water consumption [21]. Additionally, eco-feedback for fuel conservation via green transportation has been explored in the U.S. [22].

\section{Conservation in the Developing World}

A number of studies have characterized household energy requirements in developing countries such as Brazil [13, 29, 32], Nepal [39] and India [25, 29, 33, 44]. Some focus on data from the entire country $[13,29,33]$ while others focus on a particular demography of a country [2, 25, 44]. For example, Pachauri et al. established a correlation between Indian household energy requirements and various socioeconomic characteristics [33], with income level being the most important factor, followed by literacy level, household size, and age of the head of the household. Cohen et al. [13], who conducted a similar study in Brazil, found income and the mobility of the family to be most predictive of energy use, perhaps because of large daily commutes typical in Brazil. Lenzen et al. [29] compared 
such correlations in various developing and developed nations, and found that household energy requirements in developed nations are, on average, higher than developing nations, after controlling for demographic variables.

A number of qualitative studies have looked more deeply at the underlying factors driving energy use in Indian households. Indraganti et al. [25] studied apartment dwellers in Hyderabad, India, and found that thermal comfort is achieved using natural ventilation, clothing, and other adaptive behaviors such as 'moving to an airy place' and 'drinking cold water'. Kumar et al. [28] explored the adoption of more efficient appliances and light bulbs (CFLs). Vyas [44] studied Indian middle-class women who creatively adapted existing and worn-out household objects to enrich their everyday activities and found that factors such as religious belief, traditions, family intimacy, and personal hygiene influence such sustainability practices. Agrawal et al. [2] conducted a qualitative investigation exploring how rural Indians think about forest conservation to understand the relationship between the community, government and environment preservation. However, none of these works focus on understanding how resources such as water and energy are consumed and conserved among urban residential Indians.

\section{STUDY OF URBAN INDIAN HOUSEHOLDS}

Because of the relative lack of information about how urban Indian households consume and conserve energy and water, we conducted a photo-elicitation study with 11 participants [12]. The aim of our study was to elicit a detailed picture of participants' current practices and beliefs with respect to resource consumption and conservation. We sought to understand conservation practices, the motivations behind these actions and challenges faced by participants in resource management.

\section{Method}

We used a combination of word-of-mouth and snowball sampling to recruit households. We recruited participants who were either living with their families or sharing accommodations with friends. This was done to learn how other household members affect conservation behavior. We selected households that had more than 3 electrical appliances (such as TV, refrigerator, hot water heater, mixer-grinder, audio system, and washing machine, to name a few) apart from lights and electric fans. This focused us on middle and high-income urban Indian households. We met people in their homes, and ensured that the interviewer knew the local language of the participant. All participants were from urban centers in the southern part of India, primarily Bangalore and Hyderabad.

We based our design on Dillahunt et al. [15], instructing participants to "take pictures of things that relate to energy or resource use" with their mobile phone or digital camera. All participants had one or both of these. They were given a minimum of a week to complete the task. On completion, they were asked to email the photos to their interviewer.
Two authors conducted the interviews. Interview questions were primarily derived from the photos, with a focus on why the photo was taken, and its importance to conservation. Interviews lasted for 45 minutes to 1.5 hours and were conducted in English in all but one case. The participants and interviewer were both fluent in English. Participants took 6 photos on average $(s d=3)$. Although this is a relatively small number, it still sufficed to provide rich data about conservation practices. In the initial part of the interview participants were asked to describe their community and shared resources. We also asked about their interest in and knowledge of their neighbors' resource consumption. Following that, for each photo, the interviewer asked about the participant's thoughts and intents. At the end of the interview, each participant was asked to provide data about people with whom he/she interacts to share or use resources on a daily basis. All interviews were voice recorded. The interviewer took extensive notes during each interview. All interviews were transcribed: one was translated into English. Notes and transcripts were both used in our data analysis.

The interview coding and analysis was done in an iterative fashion following methods taken from informed grounded theory [41]. Transcriptions were open coded by one author. Two authors then jointly conducted selective coding to identify themes that were representative of the data and novel or important according to the literature (e.g., actions, barriers, and motivations $[11,15,45])$.

\section{Interview Demographics}

Interviews were conducted with 11 participants ( 4 female, 7 male) during the Spring of 2012. Table 1 provides key demographic data about our participants. The mean age of the participants was 32.18 years $(\mathrm{sd}=2.56)$. Eight lived in Bangalore, two in Hyderabad, and one in Chennai. All the participants had advanced degrees, with four having college degrees, and seven having post-graduate degrees. The average participant household included 3.45 people $(\mathrm{sd}=1.2)$ including children (six households), spouses (ten households) and adult relatives (four households). The household income was evenly distributed with one household earning between 1-3 L, three 4-6 L, one 7-9 L, three 10-20 L, one above $20 \mathrm{~L}$, and two unknown. These earnings are above average for urban India where the average earning is around $0.75 \mathrm{~L}$ [14].

The majority (seven) were working full-time, three were not working, and one was a student. Most participants (nine) were responsible for paying their own electricity bills. Parents paid the bills for two participants. All the participants owned a washing machine, six owned a smartphone, and five owned a car. Five participants had access to or owned a backup power source such as a generator and/or inverter, used during power cuts. The majority (nine) of them lived in rented homes, while two were homeowners. Seven lived in apartments and four lived in standalone houses (one or two-story buildings). 


\begin{tabular}{|c|c|c|c|c|c|c|c|c|c|c|}
\hline Name & Own/Rent & Household & Stuff & Power & Gender & Age & Edu. & Working & Annual Income & Location \\
\hline Diya & Rent & Couple, 2 children & $\mathrm{W}, \mathrm{C}$ & $\mathrm{E}, \mathrm{L}, \mathrm{G}$ & $\mathrm{F}$ & 37 & Post-grad & Not working & Unknown & Hyderabad \\
\hline Aditi & Rent & Couple, 2 children,parents & $\mathrm{W}, \mathrm{S}$ & $\mathrm{E}, \mathrm{L}, \mathrm{G}$ & $\mathrm{F}$ & 31 & Post-grad & Full-Time & $>20 \mathrm{~L}$ & Hyderabad \\
\hline Kumar & Own & Couple, parents & $\mathrm{W}, \mathrm{C}, \mathrm{S}$ & $\mathrm{E}, \mathrm{L}$ & $\mathrm{M}$ & 29 & College & Full-Time & $10-20 \mathrm{~L}$ & Chennai \\
\hline Raj & Rent & Couple, 2 children & W, S & $\mathrm{E}, \mathrm{L}$ & $\mathrm{M}$ & 34 & College & Full-Time & $7-9 \mathrm{~L}$ & Bangalore \\
\hline Bala & Rent & Couple & $\mathrm{W}$ & $\mathrm{E}, \mathrm{L}$ & $\mathrm{M}$ & 34 & Post-grad & Full-Time & 4-6 L & Bangalore \\
\hline Faiza & Rent & Living with a friend & $\mathrm{W}, \mathrm{S}$ & $\mathrm{E}, \mathrm{L}$ & $\mathrm{F}$ & 27 & Post-grad & Full-Time & 4-6 L & Bangalore \\
\hline John & Own & Couple, parents & $\mathrm{W}, \mathrm{C}$ & E, L & $\mathrm{M}$ & 31 & Post-grad & Full-Time & $10-20 \mathrm{~L}$ & Bangalore \\
\hline Shyam & Rent & Couple, child & W, C, S & E, L, I & $\mathrm{M}$ & 32 & College & Full-Time & Unknown & Bangalore \\
\hline Maya & Rent & Couple & $\mathrm{W}$ & $\mathrm{E}, \mathrm{L}$ & $\mathrm{F}$ & 29 & Post-grad & Looking & 4-6 L & Bangalore \\
\hline Kiran & Rent & Couple, child, brother & $\mathrm{W}, \mathrm{C}$ & $\mathrm{E}, \mathrm{L}, \mathrm{G}$ & $\mathrm{F}$ & 35 & College & Not working & $10-20 \mathrm{~L}$ & Bangalore \\
\hline Zaheer & Rent & Couple, child & $\mathrm{W}, \mathrm{S}$ & $\mathrm{E}, \mathrm{L}, \mathrm{G}$ & $\mathrm{M}$ & 30 & Post-grad & Student & $1-3 \mathrm{~L}$ & Bangalore \\
\hline
\end{tabular}

Table 1. Participant demographics. Participant names are anonymized. Own/Rent and Household describe living arrangements. Stuff indicates which large items the participant owns: car (C); smartphone (S); clothes washer (W). Power sources are electricity (E); liquid petroleum gas (L); generator (G); inverter (I). Edu. is final degree achieved; Working specifies whether the interviewee works. Annual Income is in units of Lakhs (L). One Lakh (L) is 100,000 INR, or about \$2,000.

\section{RESULTS}

Conservation was an integral part of participants' lifestyles. The conservation practices we observed were deeply integrated into daily activities and holistic in nature. Some examples were using natural light for reading instead of a lamp (Figure 1A); drying clothes outdoors; saving paper by reusing it; the use of "dissipated heat" from two pots on the stove to heat a third pot (Figure 1C); minimizing air conditioning use; unplugging devices not in active use (Figure 1D); eating and buying local goods; restricting automobile use; water conservation (Figure 1E and 1B); cleaning with non-electronic tools (brooms, hand washing clothes, etc.); and regular refrigerator maintenance (checking coils and seals).

Although some of these actions seem mundane and familiar on the surface, and each individual action may not amount to much savings, in the aggregate they represent a type of conservation that goes beyond what is found in other studies (e.g. [11, 15, 36, 45]). We define the deep conservation we observed as conservation practices that are contextually imposed, habitual (to the point of being natural and unnoticed), deeply integrated into daily activities and involving a combination of traditional practices, manual effort, and technology.

The following subsections illustrate deep conservation further by exploring conservation practices and motivations with respect to water, appliance usage, electricity and fuel. We end by summarizing participants' thoughts about the rapidly changing socio-economic context in which they must operate, and its influence on their beliefs and actions.

\section{Micro Managing Water Use}

Although water shortages were not a major issue during the months we conducted our interviews, access to clean drinking water was a concern. Water for drinking and other domestic purposes is either supplied by the government or by privately owned water tankers. Only three participants had wells on their household premise. Especially during the summer or when the Monsoon (rainy season in India) is delayed, the water supply in cities gets disrupted. Hence, urban residents face regular water shortages.
Typically, water conservation is a very low-tech activity (e.g., Figure $1 \mathrm{~B}$ and 1E). Participants manage water efficiently by carefully storing, using and re-using the available water. Water is usually stored in 20-25 liter buckets as shown in Figure 1E and re-used multiple times as it moves through "tiers" of cleanliness. As described by Raj and Kiran:

"This bucket of water is actually waste water which we get after washing the clothes. So this water can be used for flushing." Raj

"I reuse water after washing rice and vegetables for [watering] plants... water used for final round of rinsing will be almost clean. I reuse it for cleaning bathroom floors... water used for mopping the house will be used to clean the outer balcony... The residual water from the RO [reverse osmosis] water purifier will be used to clean dishes" - Kiran

Similar to actions of Raj and Kiran, Vyas [44] also found Indian middle class women reuse water after boiling potatoes and rice to clean silverware. Even though participants could afford to buy and store water, they tried not to overstock or to overuse just because they could afford it. "If you want to, you can bathe in 10 buckets [of water] also, but one to one and half buckets is enough to get clean." Shyam. Shyam and Raj also avoided wasting water at home or when out: "[before leaving a restaurant] I tend to finish it [left over drinking water is wasted otherwise]. Instead if it goes into your body, it cleanses your body." - Raj. Waste was described as a violation of Vaastu, an ancient Hindu philosophy similar to Feng Shui, "You can also read Vaastu, they say if there is a leakage in a tap [any water leak for that matter], there will be a leakage in your business also." - Shyam.

Water conservation is also seen as an act of power conservation, and can sometimes involve hi-tech solutions. In Figure 2, Maya shows an automated electricity-powered pump that avoids overflow when moving water into a cistern and thus minimizes wasted electricity.

\section{Summary}

To summarize, participants were prudent and thrifty in their use of water. This attitude probably developed over time because of water shortages. Also the high cost of drinking water, healthy living practices, and local cultural/religious traditions play a role in water conservation. The importance 
of these factors varied across participants. However, overall they represent a holistic approach to resource consumption that is concerned with more than just conservation. This holistic approach is a repeated theme in our results.

\section{Combining Manual Labor and Appliance Use}

Although much of the work around water conservation was manual in nature, the adoption of major appliances such as clothes washing machines and mixer/blenders creates a situation in which manual and automated tasks are played off against each other to save time, protect fragile clothes, and save water and energy.

\section{Washing Clothes}

Washing clothes is a daily task performed early in the day in most Indian households using a combination of hand and machine washing. This is often done by domestic staff who may help with housekeeping, clothes washing, and cooking. Even though all participants had a washing machine at home, three of them preferred to use the machine for bed linens, towels and other hardy fabrics, and wash most clothes by hand (or have a staff member wash them): "Everyday wear clothes will be less dirty and can be hand washed... It will hardly take 10-15 minutes" - Kiran. Protecting delicate cloth and cleanliness (especially on shirt collars and pant cuffs) were motivating factors for this decision. Saris and embroidered Salwar Kameez, the most common clothes worn by Indian women, can at times be damaged if washed using a washing machine [10].

Even when washing machines were used, participants employed innovative techniques to minimize electricity and water consumption. For instance, items were pre-soaked in a separate bucket of water to save energy and water by skipping the machine's soaking cycle. Two participants saved energy by skipping the spinning cycle as well.

\footnotetext{
"We generally immerse our clothes in the bucket itself... [the machine] will not consume the time in soaking part... then we set it to wash and finally, we'll just stop at the spinning part and then we'll take out our clothes." - Bala
}

Clothes dryers are rare in India, and traditionally, clothes are dried on a line. Participants did not seem concerned about the time commitment for drying clothes on a line: "this [sunlight] is natural and is free of cost, you just have to put [the clothing out] and hang it" - Bala. However, timeconstraints influenced other actions (such as washing clothes in bulk only on the weekends).

\section{Cooking}

For cooking at home, the participants again relied on manual work and automated solutions. Cutting/chopping of vegetables were usually performed manually. All except one household had a mixer/grinder system at home, which was mostly used for very specific grinding purposes, mostly when cooking in bulk was required. For day-to-day grinding, Kiran used an earthenware vessel: "I usually smash grains using this earthenware vessel. Grams and pulses can also be smashed in it instead of pressure-cooking, which helps in reducing the usage of $L P G$." - Kiran. LPG is at times scarce or unavailable, and very costly.

Participants were also still employing traditional methods for cooking and cleaning even though they had access to modern appliances. None of the participants had a vacuum cleaner. They swept floors manually using broomsticks and doing that was seen as energy efficient, and healthy: "Traditional methods, though time consuming and hard to perform are good for health and energy." - Kiran.

\section{Summary}

The conservation actions we observed were motivated by multiple factors. For instance, hand-washing clothes not only saved water and electricity, but also protected delicate clothes, while sweeping floors manually was considered to be good for health. Time was a complicated factor in all this. Rather than be concerned about time lost due to manual effort, participants spoke of health and energy gained. On the other hand, when time was short some behaviors could circumvent conservation.

\section{Electricity Use: Backups, Switches and Maintenance} In urban India, unscheduled and scheduled power cuts occur multiple times per week, sometimes lasting several hours at a time. While some apartment complexes hide the effects of this by providing backup power, most do not, and even backup power is limited to a few key plugs. When necessary, participants use their own backup solutions such as in-home batteries (with an inverter). These solutions are used only for essential loads such as night-time lighting and fans. According to Kiran, "We usually use it [the generator] when there is short power failure for at most half an hour to an hour to power one light and a fan. We also try to avoid using it during the daytime when there is enough [natural] lighting."

Two participants expressed an interest in local energy generation. However, they were unaware of local energy generation techniques (such as solar panels and micro wind turbines) that are being promoted and subsidized by the Indian government.

Participants also mentioned other strategies for efficiently managing power cuts. A few specific examples are: not opening the refrigerator (so that the internal temperature does not rise) and shifting the time of food preparation in the case of a scheduled power cut: "[We] plan the work earlier and if there is unexpected power cut, we need to alter the plans and the food items." - Maya.

Although power shortages were a strong motivator for minimizing electricity use, participants also tried to minimize their electricity consumption at other times. For example, they purchased energy efficient appliances, and avoided all possible stand-by consumption. As is common throughout India, all of the participants' power sockets have a switch as shown in Figure 1D. Participants attentively switched off these sockets when appliances plugged in were not in use. This not only saves money, but also protects appliances from power surges. 


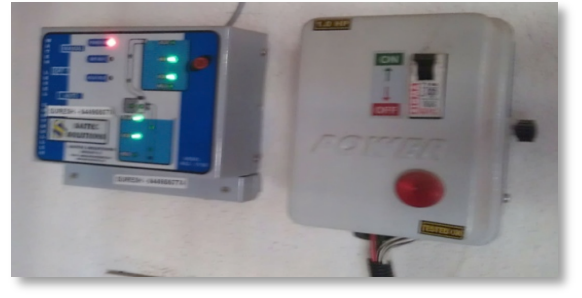

Figure 2: A water pump switch installed in Maya's home, which automatically turns on and off the pump based on the water level sensing in the overhead tank. Participants questioned the idea of monitoring energy use ("I want to save power, not monitor it"Shyam) but were willing to use technology to avoid any wastage of electricity or water ("the instrument [shown in this image] will take care of filling and there is no power wastage... [and] they maintain the water resource"-Maya)

Participants used large appliances minimally and with attention to efficient use. For example, participants used air conditioners (AC) only when they slept, and depended on ceiling fans and fresh air during other times. "Whenever I switch on the AC, I just imagine the electricity bill [going up], so I try to minimize AC use... [the air] cooler uses less energy, the bills are less, and fan is of course the best." - Aditi. Four participants pointed out that of the appliances in their home, only refrigerators consume energy all the time. Shyam and Bala mentioned that they clean the interiors and check the door seals of their refrigerators regularly to make sure they operate at maximum efficiency: "Every week we clean the refrigerator... so it closes tightly. Never leave your refrigerator open. [You] will feel the heat if you put your hand [on the] back side of the refrigerator." - Shyam.

Surprisingly, multiple participants felt that energy monitors were not an effective mechanism for reducing energy consumption. Anything that must be left on all the time is typically seen as wasteful. For example, when discussing an appliance purchase, the interviewer asked "what kind of feedback are you interested in getting ... to monitor energy." Shyam energetically stated: "Why should we bother about those [monitoring] devices.... I know how much I am consuming, how much I am paying." Participants already knew their monthly electric and water bills (perhaps because a majority paid these themselves, as mentioned earlier), and chose to invest in conservation behaviors and energyefficient appliances when it made financial sense: "Cost is one of the deciding factor that plays in all people's minds but if its longer term benefits are really good and if it is worth investing in one then definitely I would go for it" - Bala. Two participants (Kumar and Bala) preferred to purchase energy efficient appliances over energy monitors, while two other participants (Zaheer and John) felt that real-time feedback on how to reduce consumption would help them.

\section{Summary}

Resource shortages (power cuts) were only one motivation for these conservation actions. Participants also mentioned cost and waste. Practical issues such as protecting appliances from power surges and avoiding dust for health reasons also played a role in both conservation and consumption, as did personal comfort.

\section{Defraying Fuel Expenses}

Fuel in urban India is used most commonly for cooking, driving, and running generators. All participants were worried about availability of fuel in the future and increasing fuel prices: "The natural resources and then the other ancillary resources which we get from all these natural products... is very limited." - Kumar

In addition, healthy living acted as a motivator to use minimal fuel, as Faiza said, "Our mom tells us to go by walk, it's good for health, and you will be fit and fine". Despite such concerns, most participants drove regularly. Interview participants identified road safety, time, and comfort, as the main barriers in taking these green actions:

"I definitely love cycling. The primary factor why I stopped cycling these days is the [road] safety factor." - Kumar. "Time is very crucial for us... You have to walk a bit then take a bus then walk a bit and then get something else to reach your office... So, that's where we felt finally to have a two wheeler [moped/motorcycle] so that I can save my time." - Bala

Even when participants owned a vehicle, they drove it with fuel price in mind. There is a tendency to turn off vehicles (especially two-wheelers) while waiting for traffic lights to turn green. The main reason for doing so is to reduce petrol consumption as well as air pollution. The Government encourages such actions by installing traffic light timers (that display the estimated wait times) and through campaigns. "I think that's a small thing ... if it [waiting-time] is more than a minute or so, then I can switch it [the vehicle] off, and then start." - Raj. Participants also complained about the unavailability of certain alternative sources of energy. For instance, John wanted to use biomass for cooking, however "in smaller settlements like smaller towns and villages biomass is in abundance but not in cities."

\section{Summary}

Conserving fuel is a part of the daily practice of participants. As with other issues, participants had a holistic view of its value (including saving money, saving resources for the future and healthy living). However, they traded these motivations for saving off against the complexities of daily life including safety, time and availability of alternative products.

\section{Impact of a Changing Context}

As societal context influences resource consumption patterns, participants' attitudes about the changing socioeconomic context in India provides insight into additional motivational factors that may have an influence on conservation behaviors.

Participants enthusiastically described a variety of high-tech and low-tech sustainable technologies that they already used or hoped to use, such as stabilized mud blocks (a substitute to conventional bricks that requires $70 \%$ lesser energy than bricks for production [43]), a water boiler system fueled by coconut husks, solar-powered water 
heaters and street lights, and energy-efficient cookware. At the same time, participants were aware of the potential for unintended consequences of technological innovations. For instance, although Raj was excited about the prospects of using water as a fuel for cars, he was also concerned that this could lead to increased water shortages.

In spite of concerns regarding resource scarcity, participants were optimistic about the future sustainability of India. They tended to think about this in terms of the actions of individuals. While a focus on individuals has been problematized in the literature (e.g., [16]), it is nevertheless common. Kumar described the impact of a sign put up in his neighborhood: “...there was lot of garbage piling up ... over a period of time. And then someone put up this board with this beautiful saying... someone really felt that with this particular sentence over there, they wouldn't put garbage over there, and then, they cleaned it up. And then, the other people actually stopped putting garbage over there." Kumar believed that this positive impact could spread more widely. Similarly, Raj states: "One fine day, they will come to a situation, they will change. That change will encourage [them] to influence other people. It will be chain reaction." This optimism emphasizes the potential for simple motivational strategies to influence conservation behavior that focuses on individuals.

Participants were cognizant of the importance of education, stating that conservation beliefs must be "inculcated into each kid in school... [just] like [they are taught] how you brush your teeth ... It should be part of the blood" - Raj. Bala points out that such learning starts at home: "When [my nephews want] to switch on the light. [I ask] ok boss, now try to figure out where you can sit comfortably and read without turning the lights on". These beliefs are consistent with the observation that participants were motivated to conserve by what they learned at schools or from observing their parents and grandparents performing those practices.

\section{DISCUSSION}

Our study highlights a complex and adaptive set of behaviors and attitudes that demonstrate a deep commitment to conservation. For the most part, things that seem burdensome (for example, wasting time or giving up on comfort to save energy) were a natural extension of participants' culture, context, beliefs and goals. However, participants did not necessarily think about "being green" when they performed these actions. Instead, participants were motivated by a holistic set of concerns, which encompassed conservation but also goals relating to health, tradition, cost, and scarcity. In addition, participants were cognizant of the complex tradeoffs between potentially conflicting goals, and the impact of barriers such as limited available time.

In the conservation practices we observed, some key types of actions were visible across categories of conservation. Our action categories below were inspired by the action vocabulary of Pierce et al. [36], which includes cutting (powering off/down), trimming (using a "lower" setting e.g. with the thermostat), switching (using a more energy- efficient product), upgrading (acquiring a replacement product that is more efficient), and shifting use (to a different time or place). However our findings suggest additional categories, as described below.

Cutting, Trimming, and Shifting: A range of actions reduced or minimized or shifted the time of energy use. Examples include not turning on lights, not using AC even during hot times of day, using 1 instead of 10 buckets for washing, and cooking at different times due to power cuts.

Upgrading, Downgrading, and Maintaining: Technology is chosen and maintained with careful consideration of how it will influence resource use. In some cases this means choosing high tech solutions (such as the pump cistern and the AMC cookware), in other cases, it means choosing lowtech solutions such as a broom or an earthenware vessel for smashing grains. Equally important is the ongoing maintenance of technology (such as the regular cleaning of refrigerator coils).

Modularizing and Multiplying Use: From the micromanagement we saw with respect to water use, to the combination of manual and automated labor in the case of washing and kitchen work, we can see a theme of modularity. Participants broke tasks up into modular components that allowed them to more effectively manage their use of resources. Participants also found ways to multiply the values gained from that use through recycling, re-use, or creative piggybacking of use. Examples include heating milk with dissipating heat and re-using 'different tiers' of water for washing/flushing.

Participants in our study shared motivations to conserve with participants in past studies of typical $[11,36]$ and lowincome households [15] including money, comfort and religion. Barriers to conservation such as money, comfort and safety also overlapped past studies. While there is some overlap in our findings with prior studies of sustainability, there are also some key differences. Table 2 summarizes our results and compares them to studies of different demographic groups studied in North America. We highlight two differences here: the impact of resource shortages (scarcity); and the value of eco-feedback.

Scarcity: Similar to participants from low-income North American communities [15], conservation habits among our participants developed as a result of past experiences with scarcity. Perhaps as a result of this, personal preference was not as prominent in our data as in other work. For example, Pierce et al. describe participants who were "unwilling to alter their interactions [because] ... 'I need 'em ... I want 'em ', in reference to appliances like the television and air conditioner [36]. Such inflexibility, as Pierce characterizes it, is difficult to develop in environments with scarce resources.

Eco-feedback: Our participants' views of eco-feedback technologies also differed from US sustainability studies of "green" [45], and "typical" participants [11, 36]. "Green" 


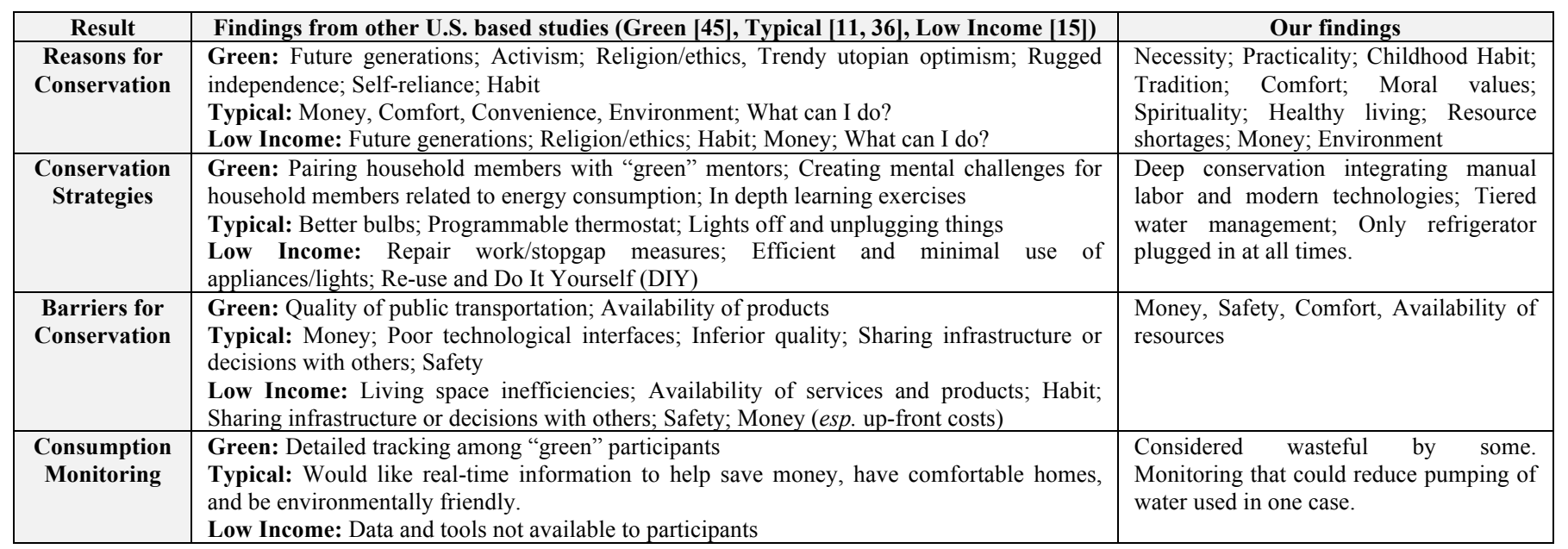

Table 2. A comparison of key findings from our work and three studies conducted in the United States.

participants leveraged eco-feedback technologies to aid in energy reduction and "typical" participants preferred monitoring devices to save money, increase comfort and decrease consumption. Our participants, however, viewed these technologies as potentially wasteful.

A limitation of our study is our small sample size. India's cultural, climate, demographic and economic diversity are hard to capture in any one sample. In our case, participants were highly educated, living with friends/family, and belonged to middle and high-income urban residential households. Additionally they were all from three cities in southern India, most from Bangalore. This population may have been at one end of a spectrum in terms of conservation behaviors. One benefit of this particular population is that they are likely to be "early adopters" of technology.

However, the wide range of behaviors, motivations, and barriers that we found provide a valuable starting place for exploring ideas that move beyond existing work on ecofeedback. For example, factors such as resource shortages, the use of household staff, and the prevalence of manual labor of different sorts in daily activity are all new to the literature on sustainability and likely to appear in other contexts. Factors such as childhood habit, healthy living, comfort, financial constraints, and safety are all present in existing literature and also the work presented in this study. While the specifics may differ across different social, economic, and cultural settings, the importance of investigating and addressing these factors in the design of eco-feedback technologies is clear from our work and that of others who have studied conservation behavior.

\section{DESIGN IMPLICATIONS}

The data we have presented provides a rich basis for rethinking the design of technology to increase resource conservation. While the population we focused on provided many insights into urban households and their conservation practices, participants also raised issues that cannot be addressed by an individual or a family. Power availability (and the management of who gets resources when); government policies relating to various types of progress; and infrastructure quality; all have a big impact on what participants could or did do. This in turn helped to highlight issues beyond resource conservation. Our design guidelines reflect on the wide ranging issues we saw with respect to the action categories described in our discussion section.

Cutting and Trimming Appliance Use: Built-in point-of-use feedback. Awareness and monitoring technologies have been a significant focus within sustainable HCI. However, our findings indicate that monitoring technologies may not be sufficient. Since participants felt they are aware of their energy use, they would rather invest in more efficient appliances than monitoring systems. Systems that provide point-of-use feedback and/or suggest efficiency measures, as proposed in [30], may resonate more with this population than ones that only provide consumption data. Such systems could be integrated into appliances so that they collect data, show feedback, and take action only when the appliance is being used.

Modularizing Use. Our findings demonstrated a strong reliance on doing things manually, even when automated solutions were available, often as a way of conserving resources. An example is that participants made use of only some cycles (rinsing, spinning, etc.) of their washing machines. Such a hybrid approach to task completion was one of the distinguishing characteristics of our participant households. Technological solutions should allow users the flexibility to have manual control over some sub-tasks. For example, a battery backup that includes a manual control for altering the charging/discharging functions as per users' energy needs would be appropriate as the lead-acid battery life and available capacity are determined by charging/discharging rates and the depth of discharge.

Opportunistic Resource Consumption in the face of Cutting and Shifting. Frequent power cuts as well as the common habit of turning off technologies when not in active use suggest it is important to consider developing technologies that are "opportunistic" and flexible in their resource 
consumption. For instance, consumer electronics appliances with local energy storage such as the Toshiba TV with integrated battery [38] do not require constant power. Similarly, Energenie [17] shuts down TV and DVD players if they are in standby mode for more than a particular duration. Of course, solutions need to be carefully evaluated in terms of conversion losses, battery lifetime and costeffectiveness, against alternatives such as regular consumer appliances with centralized battery backup solutions.

Multiplying Use by Harnessing By-Products. Participants in our study multiplied resources by making use of waste products such as dissipated heat from the stove and viewed technologies that consumed unnecessary resources negatively (as with energy monitors). New technologies designed to leverage waste products could overcome this view. For example, a refrigerator magnet with a temperature sensor could be powered by a refrigerator's waste heat (through the Peltier effect) and the same heat can be monitored to identify any malfunctioning.

Automated Shifting Enabled by Load Sensing. Appliances that can communicate with a utility could better support shifting, trimming and cutting with respect to available power, however the communication infrastructure necessary for this is not currently available in India. An alternative is adopting technologies such as the nPlug, which can sense from within the home what the current grid load levels are, and schedule appliances appropriately [24]. Such adoption could in turn help to address utility load problems in a bottom-up distributed fashion. However, policy level changes would be required to encourage widespread adoption of such technologies.

\section{CONCLUSION}

We have provided a rich description of some of the deep conservation practices taking place in urban households in southern India. We emphasize the specificity of our study here as a reminder to readers that this study is at best a first step towards characterizing conservation outside of the developed regions context.

Even within India, wide socio-economical, cultural, and demographic diversity makes it difficult to know exactly how broadly these findings generalize. For example, many common conservation behaviors found in lower-income settings were not mentioned by our participants. Examples include re-using plastic drinking bottles for storing oils instead of buying dedicated containers, packing a family of 5 or 6 onto a single moped, and washing dishes using sand, ash, or coconut husk where water is in short supply.

As highlighted recently by Dourish [16], a perspective that focuses solely on individuals is problematic for a variety of reasons. However, infrastructure-level change is a complex process that brings in many difficulties such as availability of networking and power resources necessary to support advanced communication solutions. A deeper exploration of feasible solutions that address infrastructure problems and policy is a topic for future work.

Resources such as water and energy are a matter of global concern, and the differences in how they are used across cultures, socio-economic contexts, climates, and geographies are significant and important. Our comparison with other similar studies demonstrates many similarities along with the great differences that we found. An understanding of these differences is a necessary first step toward a more global investigation of issues such as resource availability and scarcity.

\section{ACKNOWLEDGEMENTS}

We thank our reviewers for their insightful and influential comments on our work. We also thank our participants for their valuable input. This work was funded by Google, NSF IIS-0803733 and CBET-0854399.

\section{REFERENCES}

1. Abrahamse, W., Steg, L., Vlek, C., and Rothengatter, T. A review of intervention studies aimed at household energy conservation. J. of Env. Psychology, 25, 2005, 273-291.

2. Agrawal, A. Community, intimate government, and the making of environmental subjects in Kumaon, India. Current Anthropology 46, 2, 2005, 161-190.

3. Alam, M., Sathaye, J., and Barnes, D. Urban household energy use in India: Efficiency and policy implications. Energy Policy 26, 11, 1998, 885-891.

4. Amarasinghe, U., Shah, T., Turral, H., \& Anand, B. K. India's water future to 2025-2050: Business-as-usual scenario and deviations. IWMI, 2007.

5. Arroyo, E., Bonanni, L., and Selker, T. Waterbot: Exploring feedback and persuasive techniques at the sink. CHI 2005, 631-639.

6. Asian Development Bank. Benchmarking and data book of water utilities in India. 2007.

7. Can, S., Letschert, V., McNeil, M., Zhou, N., and Sathaye, J. Residential and transport energy use in India: Past trend and future outlook. Environmental Energy, 2009.

8. Central Electricity Authority. Monthly Power Supply Position. December 2012.

http://www.cea.nic.in/monthly_power_sup.html

9. Central Intelligence Agency. South Asia, India. The CIA World Factbook.

https://www.cia.gov/library/publications/the-worldfactbook/geos/in.html

10. Chavan, A.L., Gorney, D., Prabhu, B., and Arora, S. The washing machine that ate my sari: Mistakes in cross-cultural design. Interactions 16, 1, 2009, 26-31.

11. Chetty, M., Tran, D., and Grinter, R.E. Getting to green: Understanding resource consumption in the home. UbiComp 2008, 242-251.

12. Clark-IbáÑez, M. Framing the social world with photoelicitation interviews. American Behavioral Scientist 47, 12, 2004, 1507-1527. 
13. Cohen, C., Lenzen, M., and Schaeffer, R. Energy requirements of households in Brazil. Energy Policy 33, 2005, 555-562.

14. Desai, S., Dubey, A., Joshi, B. L., Sen, M., Sharif, A. and Vanneman, R. Human development in India: Challenges for a society in transition, 2010.

15. Dillahunt, T., Mankoff, J., Paulos, E., and Fussell, S. It's not all about "Green": energy use in low-income communities. Ubicomp 2009, 255-264.

16. Dourish, P. HCI and Environmental sustainability: The politics of design and the design of politics. DIS 2010, 1-10.

17. Energenie. http://www.webcitation.org/6DYzWBsxb

18. Energy Information and Administration. Country analysis and briefs. http://www.webcitation.org/6AngIeQWq

19. Ernst and Young. Riding the Wave. http://www.webcitation.org/6AmevWAIJ

20. Froehlich, J., Larson, E., Campbell, T., Haggerty, C., Fogarty, J., and Patel, S.N. HydroSense: Infrastructuremediated single-point sensing of whole-home water activity. UbiComp 2009, 235-244.

21. Froehlich, J., Findlater, L., Ostergren, M., Ramanathan, S., Peterson, J., Wragg, I., Larson, E., Fu, F., Bai, M., Patel, S.N., and Landay, J.A. The design and evaluation of prototype eco-feedback displays for fixture-level water usage data. CHI 2012, 2367-2376.

22. Froehlich, J., Dillahunt, T., Klasnja, P., Mankoff, J., Consolvo, S., Harrison, B., and Landay, J.A. UbiGreen: Investigating a mobile tool for tracking and supporting green transportation habits. CHI 2009, 1043-1052.

23. Froehlich, J., Findlater, L., and Landay, J. The design of eco-feedback technology. CHI 2010, 1999-2008.

24. Ganu, T., Seetharam, D. P., Arya, V., Kunnath, R., Hazra, J., Husain, S. A., De Silva, L. C., and Kalyanaraman, S. nPlug: A smart plug for alleviating peak loads. e-Energy 2012, 30.

25. Indraganti, M. Thermal comfort in apartments in India: Adaptive use of environmental controls and hindrances. Renewable Energy 36, 2011, 1182-1189.

26. Kappel, K. and Grechenig, T. "Show-me": Water consumption at a glance to promote water conservation in the shower. Persuasive 2009, Article 26.

27. Keirstead, J. Behavioural responses to photovoltaic systems in the UK domestic sector. Energy Policy 35, 2007, 4128-4141.

28. Kumar, A., Jain, S.K., and Bansal, N.K. Disseminating energy-efficient technologies: a case study of compact fluorescent lamps (CFLs) in India. Energy Policy 31, 3, 2003, 259-272.

29. Lenzen, M., Wier, M., Cohen, C., Hayami, H., Pachauri, S., and Schaeffer, R. A comparative multivariate analysis of household energy requirements in Australia, Brazil, Denmark, India and Japan. Energy 31, 2006, 181-207.
30. McCalley, L.T.; Midden, G.J.H. Computer based systems in household appliances: the study of ecofeedback as a tool for increasing conservation behavior. APCHI 1998, 344-349.

31. McKenzie, D., and Ray, I. Urban water supply in India: Status, reform options and possible lessons. Water Policy 11, 2009, 442-460.

32. Ordenes, M., Marinoski, D.L., Braun, P., and Ruther, $\mathrm{R}$. The impact of building-integrated photovoltaics on the energy demand of multi-family dwellings in Brazil. Energy and Buildings, 39, 2007, 629-642.

33. Pachauri, S. An analysis of cross-sectional variations in total household energy requirements in India using micro survey data. Energy Policy 32, 2004, 1723-1735.

34. Pierce, J. and Paulos, E. Beyond energy monitors: Interactions, energy, and emerging energy systems. CHI 2012, 665-674.

35. Pierce, J., Odom, W., and Blevis, E. Energy aware dwelling: a critical survey of interaction design for ecovisualizations. OZCHI 2008, 1-8.

36. Pierce, J., Schiano, D.J., and Paulos, E. Home, habits, and energy: examining domestic interactions and energy consumption. CHI 2010, 1985-1994.

37. Rao, N., Sant, G., and Rajan, S.C. An overview of Indian Energy Trends. 2009. Prayas, Energy Group, Pune, India.

38. Ricker, T. Toshiba's 32-inch power TV runs for 2 hours on integrated battery. http://www.webcitation.org/6DYzPLKwy

39. Rijal, H.B., Yoshida, H., and Umemiya, N. Investigation of the thermal comfort in Nepal. In International Symposium on Building Research and the Sustainability of the Built Environment in the Tropics, 243-262, 2002.

40. Shove, E., Lutzenhiser, L., Guy, S., Hackett, B., and Wilhite, H. Energy and social systems. Human Choice \& Climate Change 1998, Springer, 291-326.

41. Thornberg, R. Informed grounded theory. Scandinavian Journal of Educational Research, 56, 2012, 243-259.

42. UNICEF and WHO Report. Progress on Drinking Water and Sanitation - 2012 Update. http://www.webcitation.org/6AndA3pKR

43. Varanshi, S. P. Using stabilised mud._The Hindu, 9/18/2010. http://www.webcitation.org/6AmgP0Erv

44. Vyas, D. Domestic Artefacts: Sustainability in the context of Indian Middle Class. ICIC 2012, 119-128.

45. Woodruff, A., Hasbrouck, J., and Augustin. S. A bright green perspective on sustainable choices. CHI 2008, 313-322.

46. World Energy Outlook 2010. IEA. www.iea.org/Textbase/npsum/weo2010sum.pdf

47. World Population Data Sheet 2012, Population Reference Bureau, http://www.prb.org/pdf12/2012population-data-sheet_eng.pdf 